\title{
ATOMIC DECOMPOSITION OF HARDY TYPE SPACES ON CERTAIN NONCOMPACT MANIFOLDS
}

\author{
GIANCARLO MAUCERI, STEFANO MEDA AND MARIA VALLARINO
}

\begin{abstract}
In this paper we consider a complete connected noncompact Riemannian manifold $M$ with bounded geometry and spectral gap. We prove that the Hardy type spaces $X^{k}(M)$, introduced in a previous paper of the authors, have an atomic characterization. An atom in $X^{k}(M)$ is an atom in the Hardy space $H^{1}(M)$ introduced by Carbonaro, Mauceri and Meda, satisfying an "infinite dimensional" cancellation condition. As an application, we prove that the Riesz transforms of even order $\nabla^{2 k} \mathcal{L}^{-k} \operatorname{map} X^{k}(M)$ into $L^{1}(M)$.
\end{abstract}

\section{INTRODUCTION}

Suppose that $M$ is a complete connected noncompact Riemannian manifold with Ricci curvature bounded from below and positive injectivity radius. Denote by $-\mathcal{L}$ the Laplace-Beltrami operator on $M: \mathcal{L}$ is a symmetric operator on $C_{c}^{\infty}(M)$ (the space of compactly supported smooth complex-valued functions on $M$ ). Its closure is a self adjoint operator on $L^{2}(M)$ which, with a slight abuse of notation, we still denote by $\mathcal{L}$. We assume throughout that the bottom $b$ of the spectrum of $\mathcal{L}$ is strictly positive. Important examples of manifolds with these properties are nonamenable connected unimodular Lie groups equipped with a left invariant Riemannian distance, and symmetric spaces of the noncompact type with the Killing metric. It is known [CMM1, Section 8] that for manifolds with Ricci curvature bounded from below the assumption $b>0$ is equivalent to an isoperimetric property, which implies that $M$ has exponential volume growth, ergo the Riemannian measure is nondoubling.

In MMV2 we introduced a sequence $X^{1}(M), X^{2}(M), \ldots$ of new spaces of Hardy type on $M$ with the property that

$$
H^{1}(M) \supset X^{1}(M) \supset X^{2}(M), \ldots,
$$

and the sequence $Y^{1}(M), Y^{2}(M), \ldots$ of their dual spaces, and showed that these spaces may be used to obtain endpoint estimates for interesting spectral multipliers of $\mathcal{L}$, including the purely imaginary powers of $\mathcal{L}$, and the first order Riesz transform. Here $H^{1}(M)$ is the atomic Hardy space introduced in CMM1. Each of the inclusions above is proper and each of the spaces $X^{k}(M)$ is an isometric copy of $H^{1}(M)$. We refer the reader to Section 2 for the definitions of the spaces $H^{1}(M)$, $X^{k}(M)$ and $Y^{k}(M)$.

Key words and phrases. atomic Hardy space, BMO, noncompact manifolds, isoperimetric property, Riesz transforms.

Work partially supported by PRIN 2007 "Analisi Armonica". 
Since $X^{k}(M)$ is continuously included in $H^{1}(M)$, each function in $X^{k}(M)$ admits an atomic decomposition in terms of $H^{1}$-atoms (these are defined as classical Euclidean atoms [CW] St2, but their support is contained in balls of radius at most 1)). Recall that an atom $a$ in $H^{1}(M)$ must have integral 0 . This cancellation condition may also be expressed by saying that $a$ is orthogonal to the subspace of $L^{2}(M)$ of functions that are constant on the support of $a$.

E.M. Stein posed the question whether functions in $X^{k}(M)$ may be characterised as those functions in $H^{1}(M)$ that admit a decomposition in terms of atoms satisfying further cancellation conditions. The purpose of this paper is to prove such an atomic characterisation of $X^{k}(M)$ on manifolds as above satisfying, in the case where $k \geq 1$, the additional requirement that the first $k$ covariant derivatives of the Ricci tensor of $M$ be uniformly bounded.

Specifically, we say that $A$ is an $X^{k}$-atom if $A$ is an $H^{1}$-atom supported in a ball $B$ of radius at most 1 and is orthogonal in $L^{2}(B)$ to the space $Q_{B}^{k}$ of all functions $V$ in $L^{2}(M)$ such that $\mathcal{L}^{k} V$ is constant on a neighbourhood of $\bar{B}$. Note that, contrary to the classical case, the cancellation condition required for $X^{k}(M)$-atoms is expressed as orthogonality to a infinite dimensional subspace of $L^{2}(M)$. As far as we know, this is the first time that an "infinite dimensional" cancellation condition appears in the literature in connection with Hardy spaces.

An interesting and challenging problem is to prove $L^{p}(M)$ bounds for the Riesz transforms for $p$ in $(1, \infty)$ and endpoint results for $p=1$. After the pioneering works of Stein [St1] and R.S. Strichartz [Str], several contributions have appeared recently on the subject. We refer the reader to [CD, $\mathrm{ACDH}]$ and the references therein for $L^{p}(M)$ bounds. Endpoint results in the case where $\mu$ is doubling and $M$ satisfies some extra assumptions, such as appropriate on-diagonal estimate for the heat kernel or scaled Poincaré inequality have been obtained in $\mathrm{CD}, \mathrm{Ru}, \mathrm{MRu}, \mathrm{AMR}$.

To the best of our knowledge, very little is known about $L^{p}(M)$ bounds for higher order Riesz transforms. N. Lohoué [Lo proved that if $M$ is a Cartan-Hadamard manifold such that the first $2 k$ covariant derivatives of the Riemann tensor of $M$ are uniformly bounded, and the Laplace-Beltrami operator has spectral gap, then the Riesz transforms of even order $\nabla^{2 k} \mathcal{L}^{-k}$ are bounded on $L^{p}(M)$ for every $p$ in $(1, \infty)$. The atomic characterization of the spaces $X^{k}(M)$ enables us to prove, in a more general setting, an endpoint result for $\nabla^{2 k} \mathcal{L}^{-k}$ when $p=1$, namely that these operators are bounded from $X^{k}(M)$ to $L^{1}(M)$ (see Theorem 5.2). We then obtain the $L^{p}(M)$ boundedness for $p$ in $(1,2)$ by interpolation with a classical $L^{2}(M)$ result of $\mathrm{T}$. Aubin $\mathrm{Au}$. We emphasise the fact that our proof is very short and simple.

Now we briefly outline the content of this paper. In Section 2, after stating the basic geometric assumptions on the manifold $M$ and their analytic consequences, we recall the definition of the spaces $X^{k}(M)$ and their properties. In Section 3 we define the atoms in $X^{k}(M)$, we prove some of their properties and we define the atomic space $X_{\mathrm{at}}^{k}(M)$. In Section 4, we prove that $X^{k}(M)=X_{\mathrm{at}}^{k}(M)$, with equivalent norms. The argument uses a technical lemma, whose proof is rather long and is deferred to Section 6 . In Section 5 we prove the boundedness results for the Riesz transforms of even order.

We will use the "variable constant convention", and denote by $C$, possibly with sub- or superscripts, a constant that may vary from place to place and may depend on any factor quantified (implicitly or explicitly) before its occurrence, but not on factors quantified afterwards. If $\mathcal{T}$ is a bounded linear operator from the Banach 
space $A$ to the Banach space $B$, we shall denote by $\|\mathcal{T}\|_{A ; B}$ its norm. If $A=B$ we shall simply write $\|\mathcal{T}\|_{A}$ instead of $\|\mathcal{T}\|_{A ; A}$.

\section{BASIC DEFINITIONS AND BACKGROUND MATERIAL}

Suppose that $M$ is a connected $n$-dimensional Riemannian manifold of infinite volume with Riemannian measure $\mu$. Denote by Ric the Ricci tensor, by $-\mathcal{L}$ the Laplace-Beltrami operator on $M$, by $b$ the bottom of the $L^{2}(M)$ spectrum of $\mathcal{L}$, and set $\beta=\lim \sup _{r \rightarrow \infty}[\log \mu(B(o, r))] /(2 r)$. By a result of R. Brooks $b \leq \beta^{2}[\mathrm{Br}$.

Definition 2.1. We say that $M$ has $C^{\ell}$ bounded geometry if the injectivity radius is positive and the following hold:

- if $\ell=0$, then the Ricci tensor is bounded from below;

- if $\ell$ is positive, then the covariant derivatives $\nabla^{j}$ Ric of the Ricci tensor are uniformly bounded on $M$ for all $j$ in $\{0, \ldots, \ell\}$.

Basic assumptions 2.2. We make the following assumptions on $M$ :

(i) $b>0$;

(ii) $M$ has $C^{\ell}$ bounded geometry for some nonnegative integer $\ell$.

We denote by $\kappa$ the smallest positive number such that Ric $\geq-\kappa^{2}$.

Remark 2.3. It is well known that for manifolds with properties (i)-(ii) above there are positive constants $\alpha, \beta$ and $C$ such that

$$
\mu(B(p, r)) \leq C r^{\alpha} \mathrm{e}^{2 \beta r} \quad \forall r \in[1, \infty) \quad \forall p \in M,
$$

where $B(p, r)$ denotes the geodesic ball with centre $p$ and radius $r$.

Moreover, they satisfy the uniform ball size condition, i.e., for every $r>0$

$$
\inf \{\mu(B(p, r)): p \in M\}>0 \quad \text { and } \quad \sup \{\mu(B(p, r)): p \in M\}<\infty .
$$

See, for instance, CMP, where complete references are given.

Remark 2.4. By $[\mathrm{Gr}$, Section 7.5] there exists a nonnegative number $\delta$ such that the following ultracontractive estimate holds

$$
\left\|\mathrm{e}^{-t \mathcal{L}}\right\|_{1 ; 2} \leq C \mathrm{e}^{-b t} t^{-n / 4}(1+t)^{n / 4-\delta / 2} \quad \forall t \in \mathbb{R}^{+} .
$$

Clearly this implies

$$
\left\|\mathrm{e}^{-t \mathcal{L}}\right\|_{1 ; \infty} \leq C \mathrm{e}^{-b t} t^{-\delta} \quad \forall t \in[1, \infty) .
$$

We denote by $\mathcal{B}$ the family of all balls on $M$. For each $B$ in $\mathcal{B}$ we denote by $c_{B}$ and $r_{B}$ the centre and the radius of $B$ respectively. Furthermore, we denote by $c B$ the ball with centre $c_{B}$ and radius $c r_{B}$. For each scale parameter $s$ in $\mathbb{R}^{+}$, we denote by $\mathcal{B}_{s}$ the family of all balls $B$ in $\mathcal{B}$ such that $r_{B} \leq s$.

We recall the definitions of the atomic Hardy space $H^{1}(M)$ and its dual space $B M O(M)$ given in CMM1.

Definition 2.5. An $H^{1}$-atom $a$ is a function in $L^{1}(M)$ supported in a ball $B$ with the following properties:

(i) $\int_{B} a \mathrm{~d} \mu=0$

(ii) $\|a\|_{2} \leq \mu(B)^{-1 / 2}$. 
Definition 2.6. Suppose that $s$ is in $\mathbb{R}^{+}$. The Hardy space $H_{s}^{1}(M)$ is the space of all functions $g$ in $L^{1}(M)$ that admit a decomposition of the form

$$
g=\sum_{k=1}^{\infty} \lambda_{k} a_{k}
$$

where $a_{k}$ is a $H^{1}$-atom supported in a ball $B$ of $\mathcal{B}_{s}$, and $\sum_{k=1}^{\infty}\left|\lambda_{k}\right|<\infty$. The norm $\|g\|_{H_{s}^{1}}$ of $g$ is the infimum of $\sum_{k=1}^{\infty}\left|\lambda_{k}\right|$ over all decompositions (2.3) of $g$.

The vector space $H_{s}^{1}(M)$ is independent of $s$ in $\mathbb{R}^{+}$. Furthermore, given $s_{1}$ and $s_{2}$ in $\mathbb{R}^{+}$, the norms $\|\cdot\|_{H_{s_{1}}^{1}}$ and $\|\cdot\|_{H_{s_{2}}^{1}}$ are equivalent CMM1.

Notation 2.7. We shall denote the space $H_{s}^{1}(M)$ simply by $H^{1}(M)$, and we endow $H^{1}(M)$ with the norm $H_{1}^{1}(M)$.

Definition 2.8. The space $B M O(M)$ is the space of all locally integrable functions $f$ such that $N(f)<\infty$, where

$$
N(f)=\sup _{B \in \mathcal{B}_{1}} \frac{1}{\mu(B)} \int_{B}\left|f-f_{B}\right| \mathrm{d} \mu,
$$

and $f_{B}$ denotes the average of $f$ over $B$. We endow $B M O(M)$ with the "norm"

$$
\|f\|_{B M O}=N(f) .
$$

Remark 2.9. It is straightforward to check that $f$ is in $B M O(M)$ if and only if its sharp maximal function $f^{\sharp}$, defined by

$$
f^{\sharp}(x)=\sup _{B \in \mathcal{B}_{1}(x)} \frac{1}{\mu(B)} \int_{B}\left|f-f_{B}\right| \mathrm{d} \mu \quad \forall x \in M,
$$

is in $L^{\infty}(M)$. Here $\mathcal{B}_{1}(x)$ denotes the family of all balls in $\mathcal{B}_{1}$ that contain the point $x$.

The Banach dual of $H^{1}(M)$ is isomorphic to $B M O(M)$ [CMM1, Thm 5.1].

Now we recall the definition of the new Hardy spaces $X^{k}(M)$, introduced in MMV2. For every $\sigma$ in $\mathbb{R}^{+}$denote by $\mathcal{U}_{\sigma}$ the operator $\mathcal{L}(\sigma I+\mathcal{L})^{-1}$. Observe that

$$
\mathcal{U}_{\sigma}=\mathcal{I}-\sigma(\sigma I+\mathcal{L})^{-1} .
$$

It is known that $\mathcal{U}_{\sigma}$ is injective on $L^{1}(M)+L^{2}(M)$.

Definition 2.10. For each positive integer $k$ we denote by $X^{k}(M)$ the Banach space of all $L^{1}(M)$ functions $f$ such that $\mathcal{U}_{\beta^{2}}^{-k} f$ is in $H^{1}(M)$, endowed with the norm

$$
\|f\|_{X^{k}}=\left\|\mathcal{U}_{\beta^{2}}^{-k} f\right\|_{H^{1}} .
$$

Clearly $\mathcal{U}_{\beta^{2}}^{-k}$ is an isometric isomorphism between $X^{k}(M)$ and $H^{1}(M)$.

Definition 2.11. For each positive integer $k$ we denote by $Y^{k}(M)$ the Banach dual of $X^{k}(M)$.

Remark 2.12. Since $\mathcal{U}_{\beta^{2}}^{-k}$ is an isometric isomorphism between $X^{k}(M)$ and $H^{1}(M)$, its adjoint map $\left(\mathcal{U}_{\beta^{2}}^{-k}\right)^{*}$ is an isometric isomorphism between the dual of $H^{1}(M)$, i.e., $B M O(M)$, and $Y^{k}(M)$. Hence

$$
\left\|\left(\mathcal{U}_{\beta^{2}}^{-k}\right)^{*} f\right\|_{Y^{k}}=\|f\|_{B M O} .
$$


Remark 2.13. We recall the following properties of the spaces $X^{k}(M)$, proved in MMV2]:

(i) if $\sigma$ is in $\left(\beta^{2}-b, \infty\right)$, then $\mathcal{U}_{\sigma}^{k} H^{1}(M)$ agrees with $X^{k}(M)$;

(ii) $\mathcal{U}_{\beta^{2}}: X^{k-1}(M) \rightarrow X^{k}(M)$ is an isomorphism for every positive integer $k$;

(iii) $H^{1}(M) \supset X^{1}(M) \supset X^{2}(M) \supset \cdots$ with proper continuous inclusions;

(iv) if $1 / p=1-\theta / 2$, then the complex interpolation space $\left(X^{k}(M), L^{2}(M)\right)_{[\theta]}$ is $L^{p}(M)$ (this is the analogue for the spaces $X^{k}(M)$ of the celebrated result of C. Fefferman and Stein [FeS]).

\section{Special atoms}

Atoms in $X^{k}(M)$ will be $L^{2}(M)$ functions supported in a ball $B$ that satisfy a size condition analogous to that for $H^{1}$-atoms and an infinite dimensional cancellation condition, which will be expressed as orthogonality to the space of " $k$-quasiharmonic" functions on $\bar{B}$ defined below.

Definition 3.1. Suppose that $k$ is a positive integer, and that $B$ is a ball in $M$. We say that a function $V$ in $L^{2}(M)$ is k-quasi-harmonic on $\bar{B}$ if $\mathcal{L}^{k} V$ is constant (in the sense of distributions) in a neighbourhood of $\bar{B}$. We shall denote by $Q_{B}^{k}$ the space of $k$-quasi-harmonic functions on $\bar{B}$.

Remark 3.2. Observe that the following are equivalent:

(i) $V$ is in $Q_{B}^{k}$;

(ii) $V$ is in $L^{2}(M)$ and is smooth in a neighbourhood of $\bar{B}$, and $\mathcal{L}^{k} V$ is constant therein.

Indeed, if $V$ is in $Q_{B}^{k}$, then $V$ is in $L^{2}(M)$ by the definition of the space $Q_{B}^{k}$, and $\mathcal{L}^{k} V$ is a constant in the sense of distributions in a neighbourhood of $\bar{B}$. Hence $V$ is smooth on that neighbourhood by elliptic regularity.

The converse is obvious.

Observe the following inclusions, which are direct consequences of the definition of $Q_{B}^{k}$ :

$$
Q_{B}^{1} \subset Q_{B}^{2} \subset \cdots ; \quad\left(Q_{B}^{1}\right)^{\perp} \supset\left(Q_{B}^{2}\right)^{\perp} \supset \cdots
$$

For each ball $B$ in $M$ we denote by $L_{0}^{2}(B)$ the subspace of $L^{2}(M)$ consisting of all $L^{2}(M)$ functions $f$ with support contained in the ball $\bar{B}$, and satisfying $\int_{B} f \mathrm{~d} \mu=0$.

Proposition 3.3. Suppose that $k$ is a positive integer, and that $B$ is a ball in $M$. The following hold:

(i) $\left(Q_{B}^{k}\right)^{\perp}=\left\{F \in L^{2}(M): \mathcal{L}^{-k} F \in L_{0}^{2}(B)\right\}$;

(ii) $\mathcal{L}^{-k}\left(\left(Q_{B}^{k}\right)^{\perp}\right)$ is contained in $L_{0}^{2}(B) \cap \operatorname{Dom}\left(\mathcal{L}^{k}\right)$. Furthermore, functions in $\left(Q_{B}^{k}\right)^{\perp}$ have support contained in $\bar{B}$;

(iii) $\mathcal{U}_{\beta^{2}}^{-k}\left(\left(Q_{B}^{k}\right)^{\perp}\right)$ is contained in $L_{0}^{2}(B)$.

Proof. We prove (i). First we show that $\left(Q_{B}^{k}\right)^{\perp}$ is contained in $\left\{F \in L^{2}(M): \mathcal{L}^{-k} F \in L_{0}^{2}(B)\right\}$.

Suppose that $F$ is in $\left(Q_{B}^{k}\right)^{\perp}$. To show that the support of $\mathcal{L}^{-k} F$ is contained in $\bar{B}$ it suffices to prove that $\left(\mathcal{L}^{-k} F, \mathbf{1}_{B^{\prime}}\right)=0$ for every ball $B^{\prime}$ contained in $(\bar{B})^{c}$. Since $\mathcal{L}$ is self adjoint,

$$
\left(\mathcal{L}^{-k} F, \mathbf{1}_{B^{\prime}}\right)=\left(F, \mathcal{L}^{-k} \mathbf{1}_{B^{\prime}}\right) .
$$

Notice that $\mathcal{L}^{-k} \mathbf{1}_{B^{\prime}}$ is in $Q_{B}^{k}$, hence the last inner product vanishes, as required. 
Next we prove that the integral of $\mathcal{L}^{-k} F$ is 0 . Since the support of $\mathcal{L}^{-k} F$ is contained in $\bar{B}$ and $\mathcal{L}$ is self adjoint,

$$
\int_{M} \mathcal{L}^{-k} F \mathrm{~d} \mu=\left(\mathcal{L}^{-k} F, \mathbf{1}_{2 B}\right)=\left(F, \mathcal{L}^{-k} \mathbf{1}_{2 B}\right) .
$$

Now, the last inner product vanishes, because $F$ is in $\left(Q_{B}^{k}\right)^{\perp}$ by assumption and $\mathcal{L}^{-k} \mathbf{1}_{2 B}$ is in $Q_{B}^{k}$, as required.

Next we prove that $\left\{F \in L^{2}(M): \mathcal{L}^{-k} F \in L_{0}^{2}(B)\right\}$ is contained in $\left(Q_{B}^{k}\right)^{\perp}$. Suppose that $\mathcal{L}^{-k} F$ is in $L_{0}^{2}(B)$. Observe that $F$ is in $\operatorname{Dom}\left(\mathcal{L}^{k}\right)$ and that $F=\mathcal{L}^{k} \mathcal{L}^{-k} F$. Suppose now that $V$ is in $Q_{B}^{k}$. Then $V$ is smooth in a neighbourhood of $\bar{B}$ by Remark 3.2 and

$$
(F, V)=\left(\mathcal{L}^{k} \mathcal{L}^{-k} F, V\right)=\left(\mathcal{L}^{-k} F, \mathcal{L}^{k} V\right)=0 .
$$

The last equality follows from the facts that $\mathcal{L}^{k} V$ is constant in a neighbourhood of $\bar{B}$, and that $\mathcal{L}^{-k} F$ is in $L_{0}^{2}(B)$, so that its integral on $B$ vanishes.

Next we prove (ii). Clearly if $F$ is in $L^{2}(M)$, then $\mathcal{L}^{-k} F$ is in $\operatorname{Dom}\left(\mathcal{L}^{k}\right)$ by abstract set theory. Moreover $\mathcal{L}^{-k} F$ is in $L_{0}^{2}(B)$ by (i), and the first statement of (ii) follows.

To prove the second statement of (ii), observe that the support of $\mathcal{L}^{-k} F$ is contained in $\bar{B}$, hence so is the support of $\mathcal{L}^{k} \mathcal{L}^{-k} F$, i.e., of $F$.

Finally, we prove (iii). Observe that $\mathcal{U}_{\beta^{2}}^{-k}=\left(\mathcal{I}+\beta^{2} \mathcal{L}\right)^{k} \mathcal{L}^{-k}$. Since $\mathcal{L}^{-k}\left(\left(Q_{B}^{k}\right)^{\perp}\right)$ is contained in $L_{0}^{2}(B) \cap \operatorname{Dom}\left(\mathcal{L}^{k}\right)$ by (ii), it suffices to show that $\mathcal{L}^{j}\left(L_{0}^{2}(B) \cap\right.$ $\left.\operatorname{Dom}\left(\mathcal{L}^{k}\right)\right)$ is contained in $L_{0}^{2}(B)$ for all $j$ in $\{0,1, \ldots, k\}$. Suppose that $F$ is in $L_{0}^{2}(B) \cap \operatorname{Dom}\left(\mathcal{L}^{k}\right)$. Denote by $\phi$ a function in $C_{c}^{\infty}(M)$ such that $\phi=1$ on $\bar{B}$. Since $\mathcal{L}$ is self adjoint and the support of $F$ is contained in $\bar{B}$,

$$
\int_{M} \mathcal{L}^{j} F \mathrm{~d} \mu=\left(\mathcal{L}^{j} F, \phi\right)=\left(F, \mathcal{L}^{j} \phi\right)=0,
$$

as required to conclude the proof of (iii) and of the proposition.

Definition 3.4. Suppose that $k$ is a positive integer. An $X^{k}$-atom associated to the ball $B$ is a function $A$ in $L^{2}(M)$, supported in $B$, such that

(i) $A$ is in $\left(Q_{B}^{k}\right)^{\perp}$;

(ii) $\|A\|_{2} \leq \mu(B)^{-1 / 2}$.

Note that condition (i) implies that $\int_{M} A \mathrm{~d} \mu=0$, because $\mathbf{1}_{2 B}$ is in $Q_{B}^{k}$.

Remark 3.5. Note that if $A$ is a $X^{k}$-atom supported in $B$, then $\mathcal{L}^{-k} A /\left\|\mathcal{L}^{-k}\right\|_{2}$ is a $H^{1}$-atom with support contained in $\bar{B}$.

Indeed $A$ is in $\left(Q_{B}^{k}\right)^{\perp}$, so that $\mathcal{L}^{-k} A$ is in $L_{0}^{2}(B)$ by Proposition 3.3 (iii). Moreover

$$
\begin{aligned}
\left\|\mathcal{L}^{-k} A\right\|_{2} & \leq\left\|\mathcal{L}^{-k}\right\|_{2}\|A\|_{2} \\
& \leq\left\|\mathcal{L}^{-k}\right\|_{2} \mu(B)^{-1 / 2}
\end{aligned}
$$

so that $\mathcal{L}^{-k} A /\left\|\mathcal{L}^{-k}\right\|_{2}$ is a $H^{1}$-atom supported in $B$, as required.

Note also that an $X^{k}$-atom $A$ is in $X^{k}(M)$ and

$$
\|A\|_{X^{k}} \leq\left\|\mathcal{U}_{\beta^{2}}^{-k}\right\|_{2} .
$$


Indeed, the function $\mathcal{U}_{\beta^{2}}^{-k} A$ is in $L_{0}^{2}(B)$ by Proposition 3.3 (iii) and

$$
\begin{aligned}
\left\|\mathcal{U}_{\beta^{2}}^{-k} A\right\|_{2} & \leq\left\|\mathcal{U}_{\beta^{2}}^{-k}\right\|_{2}\|A\|_{2} \\
& \leq\left\|\mathcal{U}_{\beta^{2}}^{-k}\right\|_{2} \mu(B)^{-1 / 2} .
\end{aligned}
$$

Therefore $\mathcal{U}_{\beta^{2}}^{-k} A /\left\|\mathcal{U}_{\beta^{2}}^{-k}\right\|_{2}$ is an $H^{1}$-atom, and the required estimate follows from the definition of $X^{k}(M)$.

Definition 3.6. Suppose that $k$ is a positive integer. The space $X_{\mathrm{at}}^{k}(M)$ is the space of all functions $F$ in $H^{1}(M)$ that admit a decomposition of the form $F=\sum_{j} \lambda_{j} A_{j}$, where $\left\{\lambda_{j}\right\}$ is a sequence in $\ell^{1}$ and $\left\{A_{j}\right\}$ is a sequence of $X^{k}$-atoms supported in balls $B_{j}$ in $\mathcal{B}_{1}$. Atoms supported in balls in $\mathcal{B}_{1}$ will be called admissible. We endow $X_{\mathrm{at}}^{k}(M)$ with the norm

$$
\|F\|_{X_{\mathrm{at}}^{k}}=\inf \left\{\sum_{j}\left|\lambda_{j}\right|: F=\sum_{j} \lambda_{j} A_{j}, \quad A_{j} \text { admissible } X^{k} \text {-atoms }\right\} .
$$

4. The Atomic Decomposition of $X^{k}(M)$.

In this section we prove that $X^{k}(M)=X_{\mathrm{at}}^{k}(M)$ with equivalent norms. We need two lemmata.

Lemma 4.1. If $\sigma>\beta^{2}-b$ the operator $\mathcal{U}_{\sigma}^{k}$ is bounded on $H^{1}(M)$ for every positive integer $k$.

Proof. Denote by $\mathcal{D}$ the operator $\sqrt{\mathcal{L}-b}$ and by $m_{\sigma, k}$ the function defined by

$$
m_{\sigma, k}(\zeta)=\left(\frac{\zeta^{2}+b}{\zeta^{2}+b+\sigma}\right)^{k}
$$

Then $\mathcal{U}_{\sigma}^{k}=m_{\sigma, k}(\mathcal{D})$. The function $m_{\sigma, k}$ is bounded, even and holomorphic in the strip $\mathbf{S}_{\beta}=\{\zeta \in \mathbb{C}:|\operatorname{Im} \zeta|<\beta\}$ and there exists a constant $C$ such that

$$
\left|D^{j} m_{\sigma, k}(\zeta)\right| \leq C(1+|\zeta|)^{-j} \quad \forall \zeta \in \mathbf{S}_{\beta} \quad \forall j \in \mathbb{N} .
$$

The conclusion follows from [MMV2, Thm 3.4].

The main step in the proof of the atomic decomposition of $X^{k}(M)$ is Lemma 4.2 below, which will be proved in Section 6 .

Lemma 4.2. Suppose that $k$ is a positive integer and that $M$ has $C^{k}$ bounded geometry. If $A$ is an admissible $X^{k-1}$-atom then $\mathcal{U}_{4 \beta^{2}+\kappa^{2}} A$ is in $X_{\mathrm{at}}^{k}(M)$, and there exists a constant $C$, independent of $A$, such that

$$
\left\|\mathcal{U}_{4 \beta^{2}+\kappa^{2}} A\right\|_{X_{\mathrm{at}}^{k}} \leq C
$$

where $\kappa$ is the constant which appears in the lower bound of the Ricci tensor.

The main result of this section is the following.

Theorem 4.3. Suppose that $k$ is a positive integer and that $M$ has $C^{k}$ bounded geometry (see Definition 2.1). Then $X^{k}(M)$ and $X_{\mathrm{at}}^{k}(M)$ agree as vector spaces and there exists a constant $C$ such that

$$
C\|F\|_{X_{\mathrm{at}}^{k}} \leq\|F\|_{X^{k}} \leq\left\|\mathcal{U}_{\beta^{2}}^{-k}\right\|_{2}\|F\|_{X_{\mathrm{at}}^{k}} \quad \forall F \in X^{k}(M) .
$$


Proof. First we prove that $X_{\mathrm{at}}^{k}(M)$ is contained in $X^{k}(M)$, and that the right-hand inequality in (4.2) holds.

Suppose that $F=\sum_{j} \lambda_{j} A_{j}$, where $A_{j}$ is an admissible $X^{k}$-atom. By Remark 3.5 (see (3.1)), the function $\mathcal{U}_{\beta^{2}}^{-k} A_{j} /\left\|\mathcal{U}_{\beta^{2}}^{-k}\right\|_{2}$ is an $H^{1}$-atom. The series $\sum_{j} \lambda_{j} \mathcal{U}_{\beta^{2}}^{-k} A_{j}$ is then convergent in $H^{1}(M)$. Denote by $f$ its sum. Since $\mathcal{U}_{\beta^{2}}^{k}$ is bounded on $H^{1}(M)$ by Lemma 4.1

$$
\mathcal{U}_{\beta^{2}}^{k} f=\sum_{j} \lambda_{j} \mathcal{U}_{\beta^{2}}^{k}\left(\mathcal{U}_{\beta^{2}}^{-k} A_{j}\right)=F .
$$

Thus, $F$ is in $X^{k}(M)$, and

$$
\|F\|_{X^{k}}=\|f\|_{H^{1}} \leq\left\|\mathcal{U}_{\beta^{2}}^{-k}\right\|_{2} \sum_{j}\left|\lambda_{j}\right| .
$$

The right-hand inequality in (4.2) follows from this by taking the infimum over all the decompositions of $F$ of the form $F=\sum_{j} \lambda_{j} A_{j}$.

Next we prove that $X^{k}(M)$ is contained in $X_{\mathrm{at}}^{k}(M)$ and that the left-hand inequality in (4.2) holds. For notational convenience, in the rest of this proof we denote $H^{1}(M)$ also by $X^{0}(M)$, and write $\mathcal{R}$ instead of $\mathcal{R}_{4 \beta^{2}+\kappa^{2}}$ and $\mathcal{U}$ instead of $\mathcal{U}_{4 \beta^{2}+\kappa^{2}}$.

We argue inductively. The result is trivial in the case where $k=0$, because $\mathcal{U}^{0}=\mathcal{I}$. Suppose that the result holds for $k-1$ and that $F$ is in $X^{k}(M)$. Then $f=\mathcal{U}^{-1} F$ is a function in $X^{k-1}(M)$, and $\|f\|_{X^{k-1}}=\|F\|_{X^{k}}$, by Remark 2.13.

By the induction hypothesis for each $\varepsilon$ in $\mathbb{R}^{+}$there exist a sequence $\left\{A_{j}\right\}$ of admissible $X^{k-1}$-atoms and a summable sequence $\left\{c_{j}\right\}$ of complex numbers such that

$$
f=\sum_{j} c_{j} A_{j} \quad \text { and } \quad\|f\|_{X^{k-1}} \geq \sum_{j}\left|c_{j}\right|-\varepsilon .
$$

Observe that we may write

$$
F=\mathcal{U} f=\sum_{j} c_{j} \mathcal{U} A_{j}
$$

because the series $\sum_{j} c_{j} A_{j}$ converges to $f$ in $H^{1}(M)$, and $\mathcal{U}$ is bounded on $H^{1}(M)$ by Lemma 4.1 .

From (4.4) and Lemma 4.2, we see that

$$
\begin{aligned}
\|F\|_{X_{\mathrm{at}}^{k}} & \leq \sum_{j}\left|c_{j}\right|\left\|\mathcal{U} A_{j}\right\|_{X_{\mathrm{at}}^{k}} \\
& \leq C \sum_{j}\left|c_{j}\right| \\
& \leq C\left(\|f\|_{X^{k-1}}+\varepsilon\right) \\
& =C\left(\|F\|_{X^{k}}+\varepsilon\right) .
\end{aligned}
$$

Therefore $F$ is in $X_{\mathrm{at}}^{k}(M)$, and $\|F\|_{X_{\mathrm{at}}^{k}} \leq C\|F\|_{X^{k}}$, as required.

This concludes the proof of the theorem.

Remark 4.4. Suppose that $k$ is a positive integer and that $s$ is a scale parameter in $\mathbb{R}^{+}$. The space of all functions $F$ in $H^{1}(M)$ that admit a decomposition of the form $F=\sum_{j} \lambda_{j} A_{j}$, where $\left\{\lambda_{j}\right\}$ is a sequence in $\ell^{1}$ and $\left\{A_{j}\right\}$ is a sequence of $X^{k}$-atoms 
supported in balls $B_{j}$ in $\mathcal{B}_{s}$ agrees with $X_{\mathrm{at}}^{k}(M)$ (hence with $X^{k}(M)$ ). The norm on $X_{\mathrm{at}}^{k}(M)$ defined by

$$
\inf \left\{\sum_{j}\left|\lambda_{j}\right|: F=\sum_{j} \lambda_{j} A_{j}, \quad A_{j} \text { are } X^{k} \text {-atoms supported in balls of } \mathcal{B}_{s}\right\}
$$

is an equivalent norm on $X_{\mathrm{at}}^{k}(M)$.

To prove this, it suffices to observe that minor modifications in the proof of Theorem 4.3 and Lemma 4.2 show that $F$ is in $X^{k}(M)$ if and only if it admits a decomposition in terms of $X^{k}$-atoms supported in balls in $\mathcal{B}_{s}$.

Remark 4.5. Suppose that $p$ is in $(1, \infty)$ and denote by $p^{\prime}$ the index conjugate to $p$. Assume that $k$ is a positive integer and that $B$ is in $\mathcal{B}$. Define $Q_{B, p^{\prime}}^{k}$ to be the space of all functions $V$ in $L^{p^{\prime}}(M)$ such that $\mathcal{L}^{k} V$ is constant (in the sense of distributions) in a neighbourhood of $\bar{B}$. Then denote by $\left(Q_{B, p^{\prime}}^{k}\right)^{\perp}$ the annihilator of $Q_{B, p^{\prime}}^{k}$ in $L^{p}(M)$. Then a $X^{k}$-atom in $L^{p}(M)$ is an element $A$ of $\left(Q_{B, p^{\prime}}^{k}\right)^{\perp}$, satisfying the size condition

$$
\|A\|_{p} \leq \mu(B)^{-1 / p^{\prime}}
$$

It is straightforward to modify the theory of this section to show that $X^{k}(M)$ admits an atomic characterisation in terms of $X^{k}$-atoms in $L^{p}(M)$. The fact that $\mathcal{U}$ is an isomorphism of $L^{p}(M)$ for all $p$ in $(1, \infty)$ plays an important rôle here.

As a consequence of the atomic decomposition of the space $X^{k}(M)$, we may describe explicitly the action of elements of $Y^{k}(M)$, the dual of $X^{k}(M)$, on finite linear combinations of $X^{k}$-atoms.

Definition 4.6. Suppose that $k$ is a positive integer. We denote by $X_{\text {fin }}^{k}(M)$ the vector space of all finite linear combinations of $X^{k}$-atoms and by $H_{\text {fin }}^{1}(M)$ the vector space of all finite linear combinations of $H^{1}$-atoms.

Suppose that $\ell$ is a continuous linear functional on $X^{k}(M)$. Since $\mathcal{U}_{\beta^{2}}^{k}$ is an isomorphism between $H^{1}(M)$ and $X^{k}(M)$ and $X^{k}(M)$ and $X_{\mathrm{at}}^{k}(M)$ are isomorphic by Theorem 4.3, $\ell \circ \mathcal{U}_{\beta^{2}}^{k}$ is a continuous linear functional on $H^{1}(M)$. By [CMM1, Thm 5.1], there exists a function $f$ in $B M O(M)$ such that

Clearly

$$
\left(\ell \circ \mathcal{U}_{\beta^{2}}^{k}\right)(g)=\int_{M} g f \mathrm{~d} \mu \quad \forall g \in H_{\text {fin }}^{1}(M) .
$$

$$
\|\ell\|_{Y^{k}}=\left\|\ell \circ \mathcal{U}_{\beta^{2}}^{k}\right\|_{\left(H^{1}\right)^{*}}=\|f\|_{B M O} .
$$

It may be worth describing how the functional $\ell$ acts on $X^{k}$-atoms, or, more generally, on functions in $X_{\text {fin }}^{k}(M)$. Suppose that $A$ is a $X^{k}$-atom with support contained in an arbitrary ball $B$. Since $\mathcal{U}_{\beta^{2}}^{-k}=\left(\mathcal{I}+\beta^{2} \mathcal{L}^{-1}\right)^{k}$, there exist constants $c_{j}$ such that

$$
\mathcal{U}_{\beta^{2}}^{-k} A=\sum_{j=0}^{k} c_{j} \mathcal{L}^{-j} A .
$$

Then $\mathcal{U}_{\beta^{2}}^{-k} A$ is a finite linear combination of $H^{1}$-atoms by Remark 3.5 . Therefore

$$
\begin{aligned}
\ell(A) & =\left(\ell \circ \mathcal{U}_{\beta^{2}}^{k}\right)\left(\mathcal{U}_{\beta^{2}}^{-k} A\right) \\
& =\int_{M}\left(\mathcal{U}_{\beta^{2}}^{-k} A\right) f \mathrm{~d} \mu .
\end{aligned}
$$


Observe that $\mathcal{U}_{\beta^{2}}^{-k} A$ is supported in $B$, so that the last integral is just the inner product in $L^{2}(M)$ between $\mathcal{U}_{\beta^{2}}^{-k} A$ and $\mathbf{1}_{B} f$. Since $\mathcal{U}_{\beta^{2}}^{-k}$ is self adjoint, we may write

$$
\ell(A)=\int_{M} A \mathcal{U}_{\beta^{2}}^{-k}\left(\mathbf{1}_{B} f\right) \mathrm{d} \mu .
$$

A similar argument shows that if $F$ is in $X_{\text {fin }}^{k}(M)$ and its support is contained in the ball $B$, then

$$
\ell(F)=\int_{M} F \mathcal{U}_{\beta^{2}}^{-k}\left(\mathbf{1}_{B} f\right) \mathrm{d} \mu .
$$

It may be worth observing that a consequence of this representation formula for $\ell$, and of the fact that $\|\ell\|_{Y^{k}}=\|f\|_{B M O}$, is that

$$
N^{\prime}(f) \leq\|\ell\|_{Y^{k}} \leq\left\|\mathcal{U}_{\beta^{2}}^{k}\right\|_{2} N^{\prime}(f),
$$

where

$$
N^{\prime}(f)=\sup _{B \in \mathcal{B}_{1}}\left(\frac{1}{\mu(B)} \int_{B}\left|\mathcal{U}_{\beta^{2}}^{-k}\left(\mathbf{1}_{B} f\right)-f_{B} \mathcal{U}_{\beta^{2}}^{-k} \mathbf{1}_{B}\right|^{2} \mathrm{~d} \mu\right)^{1 / 2}
$$

and $f_{B}$ denotes the average of $f$ over $B$. The proof of this fact is straightforward and is omitted.

\section{RIESZ TRANSFORMS OF EVEN ORDER}

Denote by $\nabla$ the covariant derivative on $M$. The Riesz transform of order $\ell$ is the operator $\nabla^{\ell} \mathcal{L}^{-\ell / 2}$ mapping smooth functions with compact support on $M$ to sections of the bundle $T_{\ell}(M)$ of covariant tensors of order $\ell$. In this section we exploit the atomic decomposition of the spaces $X^{k}(M)$ to prove that the Riesz transforms of even order $\nabla^{2 k} \mathcal{L}^{-k}$ extend to bounded operators from $X^{k}(M)$ to the space $L^{1}\left(T_{2 k}(M)\right)$ of $L^{1}$ sections of $T_{2 k}(M)$. To prove this result we need to strengthen the bounded geometry assumption on $M$, by replacing the derivatives of the Ricci tensor with those of the Riemann tensor in Definition 2.1.

Definition 5.1. We say that $M$ has $C^{\ell}$ strongly bounded geometry if the injectivity radius is positive and the following hold:

- if $\ell=0$, then the Ricci tensor is bounded from below Ric $\geq-\kappa^{2}$ for some positive $\kappa$;

- if $\ell$ is positive, then the covariant derivatives $\nabla^{j} R$ of the Riemann tensor are uniformly bounded on $M$ for all $j$ in $\{0, \ldots, \ell\}$.

We recall that the boundedness of the first order Riesz transform on $L^{2}(M)$ follows from the identity $\mathcal{L}=\nabla^{*} \nabla$ and the self-adjointness of $\mathcal{L}$ on $L^{2}(M)$ Str. From a result of Aubin [Au. Prop. 3], it follows also that if $b>0$ and $M$ has $C^{\ell-2}$ strongly bounded geometry then the Riesz transform of order $\ell \geq 2$ extends to a bounded operator from $L^{2}(M)$ to the space $L^{2}\left(T_{\ell}(M)\right)$ of square integrable sections of $T_{\ell}(M)$.

In MMV2 the authors, under the additional assumption that $b=\beta^{2}$, proved that the Riesz transform of order 1 maps $X^{k}(M)$ to $L^{1}\left(T_{1}(M)\right)$ for $k$ large enough. In general, the Riesz transforms of order one do not map $H^{1}(M)$ to $L^{1}\left(T_{\ell}(M)\right)$. A counterexample on noncompact symmetric spaces will appear in a forthcoming paper of the authors MMV3. Notice that the modified Riesz transform of order 1, i.e. the operator $\nabla(\mathcal{L}+\varepsilon I)^{-1 / 2}$, for $\varepsilon>0$, maps $H^{1}(M)$ into $L^{1}\left(T_{1}(M)\right)$ even when $M$ satisfies less stringent assumptions on $M[\mathrm{Ru}$. 
Theorem 5.2. If $b>0$ and $M$ has $C^{2 k-2}$ strongly bounded geometry then the Riesz transform of order $2 k$ extends to a bounded operator from $X^{k}(M)$ to $L^{1}\left(T_{2 k}(M)\right)$ and from $L^{p}(M)$ to $L^{p}\left(T_{2 k}(M)\right)$ for all $p$ in $(1,2)$.

Proof. To prove that $\nabla^{2 k} \mathcal{L}^{-k}$ is bounded from $X^{k}(M)$ to $L^{1}\left(T_{2 k}(M)\right)$ it suffices to show that $\nabla^{2 k} \mathcal{L}^{-k}$ maps $X^{k}$-atoms into $L^{1}\left(T_{2 k}(M)\right)$ uniformly. Suppose that $A$ is a $X^{k}$-atom associated to the ball $B$. Then, by Remark 3.5, the function $\mathcal{L}^{-k} A$ is supported in $\bar{B}$. Hence, by Hölder's inequality and the boundedness of $\nabla^{2 k} \mathcal{L}^{-k}$ on $L^{2}(M)$,

$$
\begin{aligned}
\left\|\nabla^{2 k} \mathcal{L}^{-k} A\right\|_{1} & \leq\left\|\nabla^{2 k} \mathcal{L}^{-k} A\right\|_{2} \mu(B)^{1 / 2} \\
& \leq C\|A\|_{2} \mu(B)^{1 / 2} \leq C,
\end{aligned}
$$

as required. The boundedness of $\nabla^{2 k} \mathcal{L}^{-k}$ from $L^{p}(M)$ to $L^{p}\left(T_{2 k}(M)\right)$ for all $p$ in $(1,2)$ follows by interpolation (see MMV2, Thm 2.15]) from the $X^{k}(M)-$ $-L^{1}\left(T_{2 k}(M)\right)$ boundedness and the aforementioned result of Aubin.

\section{Proof of Lemma 4.2}

In this section we shall prove Lemma 4.2. First we need a variant of the "economical decomposition of atoms" proved in [MMV2, Lemma 5.7].

Lemma 6.1. Suppose that $k$ is a positive integer and that $M$ has $C^{k}$ bounded geometry (see Definition 2.1). If a is an $H^{1}$-atom in $\operatorname{Dom}\left(\mathcal{L}^{k}\right)$, then $\mathcal{L}^{k} a$ is in $X_{\mathrm{at}}^{k}(M)$. Furthermore, if the support of a is contained in the ball $B$, then there exists a constant $C$ such that

$$
\left\|\mathcal{L}^{k} a\right\|_{X_{\mathrm{at}}^{k}} \leq C\left(1+r_{B}\right) \mu(B)^{1 / 2}\left\|\mathcal{L}^{k} a\right\|_{2} .
$$

Proof. Suppose first that the support of $a$ is contained in a ball $B$ such that $r_{B} \leq 1$. Since $\mathcal{L}^{k} a$ is in $\left(Q_{B}^{k}\right)^{\perp}$ by Proposition 3.3, $\mu(B)^{-1 / 2} \mathcal{L}^{k} a /\left\|\mathcal{L}^{k} a\right\|_{2}$ is a $X^{k}$-atom supported in a ball in $\mathcal{B}_{1}$ and the lemma is proved.

Next, suppose that $r_{B}>1$. Denote by $\mathfrak{S}$ a $1 / 3$-discretisation of $M$, i.e. a set of points in $M$ that is maximal with respect to the property

$$
\min \{d(z, w): z, w \in \mathfrak{S}, z \neq w\}>1 / 3, \quad \text { and } \quad d(\mathfrak{S}, x) \leq 1 / 3 \quad \forall x \in M .
$$

The family $\{B(z, 1): z \in \mathfrak{S}\}$ is a covering of $M$ which is uniformly locally finite, by the uniform ball size and the local doubling property of the Riemannian measure (see, for instance, [Ch Theorem 3.10]). By the same token, the set $B \cap \mathfrak{S}$ is finite and has at most $N$ points $z_{1}, \ldots, z_{N}$, with $N \leq C \mu(B)$, where $C$ is a constant which does not depend on $B$. Denote by $B_{j}$ the ball with centre $z_{j}$ and radius 1 , and by $\left\{\psi_{j}: j=1, \ldots, N\right\}$ a partition of unity on $B$ subordinated to the covering $\left\{B_{j}: j=1, \ldots, N\right\}$.

Fix $j$ in $\{1, \ldots, N\}$ and denote by $z_{j}^{0}, \ldots, z_{j}^{N_{j}}$ points on a minimizing geodesic joining $z_{j}$ and $c_{B}$, with the property that $z_{j}^{0}=z_{j}, z_{j}^{N_{j}}=c_{B}$, and $d\left(z_{j}^{h}, z_{j}^{h+1}\right)$ is approximately equal to $1 / 3$. Note that $N_{j} \leq 4 r_{B}$. Denote by $B_{j}^{h}$ the ball $B\left(z_{j}^{h}, 1 / 12\right)$, for $j=1, \ldots, N$ and $h=0, \ldots, N_{j}$. Then the balls $B_{j}^{h}$ are disjoint, $B_{j}^{h} \subset B\left(z_{j}^{h}, 1\right) \cap B\left(z_{j}^{h+1}, 1\right)$ and $B_{j}^{N_{j}}=B\left(c_{B}, 1 / 12\right)$.

Denote by $\phi_{j}^{h}$ a nonnegative function in $C_{c}^{\infty}\left(B_{j}^{h}\right)$ that has integral 1. By the uniform ball size property we may choose the functions $\phi_{j}^{h}$ so that there exists a constant $A$ such that $\left\|\phi_{j}^{h}\right\|_{2} \leq A$ for all $h$ and $j$. 
The existence of a uniform bound on the derivatives of the Ricci tensor implies that we can choose the functions $\psi_{j}$ and $\phi_{j}^{h}$ so that their covariant derivatives of order up to $2 k$ are uniformly bounded for all $j$ and $h$ (see [He, p. 14]). Now, denote by $a_{j}^{0}$ the function $a \psi_{j}$. Clearly

$$
a=\sum_{j=1}^{N} \psi_{j} a=\sum_{j=1}^{N} a_{j}^{0}
$$

Next, define

$$
a_{j}^{1}=a_{j}^{0}-\phi_{j}^{0} \int_{M} a_{j}^{0} \mathrm{~d} \mu \quad \text { and } \quad a_{j}^{h}=\left(\phi_{j}^{h-2}-\phi_{j}^{h-1}\right) \int_{M} a_{j}^{0} \mathrm{~d} \mu, \quad 2 \leq h \leq N_{j}+1 .
$$

Then, for every $h$ in $\left\{1, \ldots, N_{j}\right\}$, the support of $a_{j}^{h}$ is contained in $B\left(z_{j}^{h-1}, 1\right)$, the integral of $a_{j}^{h}$ vanishes and

$$
\begin{aligned}
\left\|a_{j}^{h}\right\|_{2} & \leq 2 A \int_{M}\left|a_{j}^{0}\right| \mathrm{d} \mu \\
& \leq C\left\|a_{j}^{0}\right\|_{2} \mu\left(B_{j}\right)^{1 / 2} \\
& \leq C\left\|a_{j}^{0}\right\|_{2} \mu\left(B_{j}^{h}\right)^{-1 / 2} .
\end{aligned}
$$

In the last two inequalities we have used the uniform ball size property (2.2). Hence there exists a constant $C$, independent of $j$ and $h$, such that

$$
\left\|a_{j}^{h}\right\|_{H^{1}} \leq C\left\|a_{j}^{0}\right\|_{2} \text {. }
$$

Moreover

$$
a_{j}^{0}=\sum_{h=1}^{N_{j}+1} a_{j}^{h}+\phi_{j}^{N_{j}} \int_{M} a_{j}^{0} \mathrm{~d} \mu
$$

Thus

$$
a=\sum_{j=1}^{N} \sum_{h=1}^{N_{j}+1} a_{j}^{h}
$$

because $\sum_{j} \int_{M} a_{j}^{0} \mathrm{~d} \mu=\int_{M} a \mathrm{~d} \mu=0$ and all the functions $\phi_{j}^{N_{j}}, j=1, \ldots, N_{j}$ coincide, since $B_{j}^{N_{j}}=B\left(c_{B}, 1 / 12\right)$. Moreover, $a_{j}^{1}$ is in $\operatorname{Dom}\left(\mathcal{L}^{k}\right)$, and

$$
\begin{aligned}
\left\|\mathcal{L}^{k} a_{j}^{1}\right\|_{2} & \leq\left\|\mathcal{L}^{k} a_{j}^{0}\right\|_{2}+\left\|\mathcal{L}^{k} \phi_{j}^{0}\right\|_{2} \int_{M}\left|a_{j}^{0}\right| \mathrm{d} \mu \\
& \leq C\left\|\mathcal{L}^{k} a_{j}^{0}\right\|_{2},
\end{aligned}
$$

where, in the last inequality, we have used the estimate $\left\|a_{j}^{0}\right\|_{2} \leq C\left\|\mathcal{L}^{k} a_{j}^{0}\right\|_{2}$, which holds because $\mathcal{L}$ has spectral gap. Similarly, if $h=2, \ldots, N_{j}+1$, then $a_{j}^{h}$ is in $\operatorname{Dom}\left(\mathcal{L}^{k}\right)$, and

$$
\left\|\mathcal{L}^{k} a_{j}^{h}\right\|_{2} \leq C\left\|\mathcal{L}^{k} a_{j}^{0}\right\|_{2}
$$

Hence $\mathcal{L}^{k} a_{j}^{h} /\left\|\mathcal{L}^{k} a_{j}^{0}\right\|_{2}$ is a multiple of a $X^{k}$-atom supported in a ball of radius 1 , with a constant $C$ which does not depend on $j$ and $h$ by the uniform ball size property. Thus

$$
\left\|\mathcal{L}^{k} a_{j}^{h}\right\|_{X_{\mathrm{at}}^{k}} \leq C\left\|\mathcal{L}^{k} a_{j}^{0}\right\|_{2} \quad \forall j, h
$$


Adding up the inequalities in (6.2), we obtain

$$
\begin{aligned}
\left\|\mathcal{L}^{k} a\right\|_{X_{\mathrm{at}}^{k}} & \leq \sum_{j=1}^{N} \sum_{h=1}^{N_{j}+1}\left\|\mathcal{L}^{k} a_{j}^{h}\right\|_{X_{\mathrm{at}}^{k}} \\
& \leq C \sum_{j=1}^{N} \sum_{h=1}^{N_{j}+1}\left\|\mathcal{L}^{k} a_{j}^{0}\right\|_{2} .
\end{aligned}
$$

Remembering that $N_{j} \leq C r_{B}$ and $N \leq C \mu(B)$, and using Schwarz's inequality, we see that the right-hand side is dominated by

$$
\begin{aligned}
C r_{B} \sum_{j=1}^{N}\left\|\mathcal{L}^{k} a_{j}^{0}\right\|_{2} & \leq C r_{B} N^{1 / 2}\left(\sum_{j=1}^{N}\left\|\mathcal{L}^{k} a_{j}^{0}\right\|_{2}^{2}\right)^{1 / 2} \\
& \leq C r_{B} \mu(B)^{1 / 2}\left\|\mathcal{L}^{k} a\right\|_{2} .
\end{aligned}
$$

In the last inequality we have used the fact that $\left\{\psi_{j}\right\}$ is a partition of unity on $B$, subordinated to the uniformly locally finite covering $\left\{B_{j}\right\}$.

This completes the proof of the lemma.

Remark 6.2. There exists a constant $C$ such that

$$
\|f\|_{X_{\mathrm{at}}^{k}} \leq C\left(1+r_{B}\right) \mu(B)^{1 / 2}\|f\|_{2} \quad \forall f \in\left(Q_{B}^{k}\right)^{\perp} .
$$

Indeed, if $f$ is in $\left(Q_{B}^{k}\right)^{\perp}$, then the function $\mathcal{L}^{-k} f$ is a multiple of a $H^{1}$-atom, by Proposition 3.3 The conclusion follows, by Lemma 6.1

The second ingredient in our proof of Lemma 4.2 are two technical results in onedimensional Fourier analysis (see Lemma6.3 and Lemma 6.5 below). To state them we need some more notation. For every $f$ in $L^{1}(\mathbb{R})$ define its Fourier transform $\widehat{f}$ by

$$
\widehat{f}(t)=\int_{-\infty}^{\infty} f(s) \mathrm{e}^{-i s t} \mathrm{~d} s \quad \forall t \in \mathbb{R} .
$$

If $f$ is a function on $\mathbb{R}$, and $\lambda$ is in $\mathbb{R}^{+}$, we denote by $f^{\lambda}$ and $f_{\lambda}$ the $\lambda$-dilates of $f$, defined by

$$
f^{\lambda}(x)=f(\lambda x) \quad \text { and } \quad f_{\lambda}(x)=\lambda^{-1} f(x / \lambda) \quad \forall x \in \mathbb{R} .
$$

For each $\nu \geq-1 / 2$, denote by $\mathcal{J}_{\nu}: \mathbb{R} \backslash\{0\} \rightarrow \mathbb{C}$ the modified Bessel function of order $\nu$, defined by

$$
\mathcal{J}_{\nu}(t)=\frac{J_{\nu}(t)}{t^{\nu}}
$$

where $J_{\nu}$ denotes the standard Bessel function of the first kind and order $\nu$ (see, for instance, [L, formula (5.10.2), p. 114] for the definition). Recall that

$$
\mathcal{J}_{-1 / 2}(t)=\sqrt{\frac{2}{\pi}} \cos t \quad \text { and that } \quad \mathcal{J}_{1 / 2}(t)=\sqrt{\frac{2}{\pi}} \frac{\sin t}{t} .
$$

For each positive integer $\ell$, we denote by $\mathcal{O}^{\ell}$ the differential operator $t^{\ell} D^{\ell}$ on the real line. For the proof of the following lemma, see [MMV2, Lemma 4.1].

Lemma 6.3. For every positive integer $k$ there exists a polynomial $P_{k+1}$ of degree $k+1$ without constant term, such that

$$
\int_{-\infty}^{\infty} f(t) \cos (v t) \mathrm{d} t=\int_{-\infty}^{\infty} P_{k+1}(\mathcal{O}) f(t) \mathcal{J}_{k+1 / 2}(t v) \mathrm{d} t
$$


for all functions $f$ such that $\mathcal{O}^{\ell} f \in L^{1}(\mathbb{R}) \cap C_{0}(\mathbb{R})$ for all $\ell$ in $\{0,1, \ldots, k+1\}$.

Denote by $\omega$ an even function in $C_{c}^{\infty}(\mathbb{R})$ which is supported in $[-3 / 4,3 / 4]$, is equal to 1 in $[-1 / 4,1 / 4]$, and satisfies

$$
\sum_{j \in \mathbb{Z}} \omega(t-j)=1 \quad \forall t \in \mathbb{R} .
$$

Denote by $\phi$ the function $\omega^{1 / 4}-\omega$, where $\omega^{1 / 4}$ denotes the 1/4-dilate of $\omega$. Then $\phi$ is smooth, even and vanishes in the complement of the set $\{t \in \mathbb{R}: 1 / 4 \leq|t| \leq 4\}$. For a fixed $R$ in $(0,1]$ and for each positive integer $i$, denote by $E_{i}$ the set $\{t \in \mathbb{R}$ : $\left.4^{i-1} R \leq|t| \leq 4^{i+1} R\right\}$. Clearly $\phi^{1 /\left(4^{i} R\right)}$ is supported in $E_{i}$, and $\sum_{i=1}^{\infty} \phi^{1 /\left(4^{i} R\right)}=1$ in $\mathbb{R} \backslash(-R, R)$. Denote by $d$ the integer $\left[\log _{4}(3 / R)\right]+1$. To avoid cumbersome notation, we write $\rho_{i}$ instead of $1 /\left(4^{i} R\right)$. Then

$$
\omega^{\rho_{0}}+\sum_{i=1}^{d} \phi^{\rho_{i}}=1 \quad \text { on } \quad[-3,3] .
$$

Suppose that $c$ is in $\mathbb{R}^{+}$, and denote by $r$ the function defined by

$$
r(\lambda)=\frac{1}{c^{2}+\lambda^{2}} \quad \forall \lambda \in \mathbb{C} \backslash\{ \pm i c\} .
$$

Note the decomposition

$$
\widehat{\omega} * r(\lambda)=\sum_{i=0}^{d} S_{i}(\lambda),
$$

where the functions $S_{i}: \mathbb{R} \rightarrow \mathbb{C}$ are defined by

$$
S_{0}(\lambda)=\frac{1}{2 \pi} \int_{-\infty}^{\infty} \omega^{\rho_{0}}(t) P_{N}(\mathcal{O})(\omega \widehat{r})(t) \mathcal{J}_{N-1 / 2}(\lambda t) \mathrm{d} t \quad \forall \lambda \in \mathbb{R}
$$

and, for $i$ in $\{1, \ldots, d\}$,

$$
S_{i}(\lambda)=\frac{1}{2 \pi} \int_{-\infty}^{\infty} \phi^{\rho_{i}}(t) P_{N}(\mathcal{O})(\omega \widehat{r})(t) \mathcal{J}_{N-1 / 2}(\lambda t) \mathrm{d} t \quad \forall \lambda \in \mathbb{R},
$$

where $N$ is a positive integer.

Remark 6.4. Note that there exist constants $c_{\ell}$ such that

$$
t^{-1} P_{N}(\mathcal{O})=\sum_{\ell=0}^{N-1} c_{\ell} t^{\ell} D^{\ell+1} .
$$

Lemma 6.5. Suppose that $N$ is a positive integer. The following hold:

(i) the norm $\left\|t^{-1} P_{N}(\mathcal{O}) \widehat{r}\right\|_{\infty}$ is finite;

(ii) if $N \geq 3$, then there exists a constant $C$, independent of $R$ in $(0,1]$, such that

$$
\sup _{\lambda \geq 0}\left(\lambda^{2}+1\right)\left|S_{0}(\lambda)\right| \leq C .
$$

Proof. By Remark 6.4, to prove (i) it suffices to show that

$$
\sup _{t \in \mathbb{R}}\left|t^{\ell} D^{\ell+1} \widehat{r}(t)\right|<\infty \quad \forall \ell \in\{0, \ldots, N-1\} .
$$


This is a standard estimate in Fourier analysis. Recall that $\widehat{r}(t)=(1 / c) \mathrm{e}^{-c|t|}$. It is straightforward to check that $D \widehat{r}=-c \widehat{r}$ sgn, and that for each $k \geq 1$

$$
\begin{aligned}
D^{2 k} \widehat{r} & =c^{2 k} \widehat{r}-2 \sum_{j=0}^{k-1} c^{2(k-1-j)} D^{2 j} \delta_{0} \\
D^{2 k+1} \widehat{r} & =-c^{2 k+1} \widehat{r} \cdot \operatorname{sgn}-2 \sum_{j=0}^{k-1} c^{2(k-1-j)} D^{2 j+1} \delta_{0} .
\end{aligned}
$$

Hence

$$
t^{2 k-1} D^{2 k} \widehat{r}(t)=c^{2 k} t^{2 k-1} \widehat{r}(t) \quad \text { and } \quad t^{2 k} D^{2 k+1} \widehat{r}(t)=-c^{2 k+1} t^{2 k} \operatorname{sgn}(t) \cdot \widehat{r}(t),
$$

so that

$$
\left|t^{\ell} D^{\ell+1} \widehat{r}(t)\right|=c^{\ell}|t|^{\ell} \mathrm{e}^{-c|t|} \quad \forall t \in \mathbb{R},
$$

and the required estimate follows.

To prove (ii), observe that, on the one hand, by (6.8) and (6.10)

$$
\begin{aligned}
\left|S_{0}(\lambda)\right| & \leq\|\omega\|_{\infty}\left\|t^{-1} P_{N}(\mathcal{O})(\omega \widehat{r})\right\|_{\infty} \int_{-\infty}^{\infty}|t|\left|\mathcal{J}_{N-1 / 2}(t \lambda)\right| \mathrm{d} t \\
& \leq C\|\omega\|_{\infty} \lambda^{-2} \quad \forall \lambda \in[0, \infty) .
\end{aligned}
$$

On the other hand, the function $\mathcal{J}_{N-1 / 2}$ is bounded, so that

$$
\begin{aligned}
\left|S_{0}(\lambda)\right| & \leq C\left\|t^{-1} P_{N}(\mathcal{O})(\omega \widehat{r})\right\|_{\infty} \int_{-\infty}^{\infty} \omega^{\rho_{0}}(t)|t| \mathrm{d} t \\
& \leq C \quad \forall \lambda \in[0, \infty) .
\end{aligned}
$$

We have used the fact that $\rho_{0}=1 / R$ and $R \leq 1$ in the last inequality. The proof of the lemma is complete.

The third, and last, ingredient in the proof of Lemma 4.2 is the following proposition, which shows that certain functions of the operator $\mathcal{L}$ map $H^{1}$-atoms into functions that have integral 0 . For technical reasons, it is convenient to work with functions of the wave propagator

$$
\mathcal{D}_{1}=\sqrt{\mathcal{L}-b+\kappa^{2}}
$$

instead of functions of $\mathcal{L}$. We recall that $-\kappa^{2}$ is the greatest lower bound of the Ricci curvature (see Basic assumptions 2.2). The reason for considering the operator $\mathcal{D}_{1}$ instead of $\mathcal{D}=\sqrt{\mathcal{L}-b}$ is that, in order to prove estimates of the gradient of the kernels associated to functions of $\mathcal{L}$, we need to exploit the identity $\mathrm{d} \mathcal{L}=\mathbb{L} \mathrm{d}$, where $\mathbb{L}$ is the Hodge Laplacian $\mathbb{L}$ on 1 -forms and d denotes exterior differentiation (see MMV1, Prop. 5.5]). Whereas, in general, the operator $\mathbb{L}-b$ is not a positive operator on 1 -forms, the operator $\mathbb{L}-b+\kappa^{2}$ is nonnegative on manifolds whose Ricci curvature satisfies the lower bound Ric $\geq-\kappa^{2}$.

Proposition 6.6. Suppose that $\nu$ is in $[-1 / 2, \infty)$, that $w$ is a complex measure on $\mathbb{R}$ and that $a$ is an $H^{1}$-atom. Define the operator $\mathcal{W}_{\nu}\left(\mathcal{D}_{1}\right)$ on $L^{2}(M)$ spectrally by

$$
\mathcal{W}_{\nu}\left(\mathcal{D}_{1}\right) f=\int_{-\infty}^{\infty} \mathcal{J}_{\nu}\left(t \mathcal{D}_{1}\right) f \mathrm{~d} w(t) \quad \forall f \in L^{2}(M) .
$$

The following hold:

(i) $\int_{M} \mathcal{W}_{\nu}\left(\mathcal{D}_{1}\right) a \mathrm{~d} \mu=0$; 
(ii) $\int_{M} S_{i}\left(\mathcal{D}_{1}\right) a \mathrm{~d} \mu=0$ for $i=0, \ldots, d$ (the functions $S_{i}$ are defined in (6.8) and (6.9).

Proof. A simple argument, based on the finite speed of propagation property of the operator $\mathcal{L}-b+\kappa^{2}$, shows that

$$
\int_{M} \mathcal{J}_{\nu}\left(t \mathcal{D}_{1}\right) a \mathrm{~d} \mu=0
$$

(see MMV2, Prop. 5.5]). Since $\mathcal{W}_{\nu}$ and the function $S_{i}$ are integrals of $\mathcal{J}_{\nu}(t \cdot)$ with respect to complex measures, we obtain the desired conclusion by interchanging the order of integration.

Remark 6.7. Note that for every $\nu$ in $[-1 / 2, \infty)$ the function $\lambda \mapsto \mathcal{J}_{\nu}(t \lambda)$ is even and of entire of exponential type $t$, so that kernel $k_{\mathcal{J}_{\nu}\left(t \mathcal{D}_{1}\right)}$ of the operator $\mathcal{J}_{\nu}\left(t \mathcal{D}_{1}\right)$ is supported in the set $\{(x, y) \in M \times M: d(x, y) \leq t\}$ by the finite propagation speed.

The main step in the proof of our main result is Lemma 4.2 which we restate for the reader's convenience. The idea, used in the proof, of subordinating spectral functions of $\mathcal{L}$ to the wave propagator has been used several times since its appearance in CGT, Ta].

Lemma (4.2). Suppose that $k$ is a positive integer and that $M$ has $C^{k}$ bounded geometry. Let $A$ be an admissible $X^{k-1}$-atom. Then $\mathcal{U}_{4 \beta^{2}+\kappa^{2}} A$ is in $X_{\mathrm{at}}^{k}(M)$, and there exists a constant $C$, independent of $A$, such that

$$
\left\|\mathcal{U}_{4 \beta^{2}+\kappa^{2}} A\right\|_{X_{\mathrm{at}}^{k}} \leq C .
$$

Proof. Suppose that the atom $A$ is supported in the ball $B(p, R)$. Then $R \leq 1$, because $A$ is admissible. Denote by $N$ an integer $>n / 2+3$. For notational convenience, in this proof we shall write $\mathcal{J}$ instead of $\mathcal{J}_{N-1 / 2}, \mathcal{R}$ instead of $\mathcal{R}_{4 \beta^{2}+\kappa^{2}}$, $\mathcal{U}$ instead of $\mathcal{U}_{4 \beta^{2}+\kappa^{2}}$ and $c$ instead of $\sqrt{4 \beta^{2}+b}$. Observe that $\mathcal{R}=r\left(\mathcal{D}_{1}\right)$ (the function $r$ was defined in (6.6)).

Step I: splitting of the operator. Define the operators $\mathcal{S}$ and $\mathcal{T}$ spectrally by

$$
\mathcal{S}=(\widehat{\omega} * r)\left(\mathcal{D}_{1}\right) \quad \text { and } \quad \mathcal{T}=(r-\widehat{\omega} * r)\left(\mathcal{D}_{1}\right) .
$$

Then $\mathcal{U} A=\mathcal{L} \mathcal{R} A=\mathcal{L} \mathcal{S} A+\mathcal{L} \mathcal{T} A$. We shall prove that both $\mathcal{L S} A$ and $\mathcal{L} \mathcal{T} A$ are in $X_{\mathrm{at}}^{k}(M)$ and that there exists a constant $C$, independent of $A$, such that

$$
\|\mathcal{L S} A\|_{X_{\mathrm{at}}^{k}} \leq C \quad \text { and } \quad\|\mathcal{L} \mathcal{T} A\|_{X_{\mathrm{at}}^{k}} \leq C .
$$

The proof of estimates (6.13) will be given in Steps II and III.

Step II: proof of the first inequality in 6.13). Note that $\omega \widehat{r}$ has support in $[-3 / 4,3 / 4]$. Define the functions $S_{i}$ as in (6.8) and (6.9). Observe that, by (6.7),

$$
\mathcal{S}=\sum_{i=0}^{d} S_{i}\left(\mathcal{D}_{1}\right),
$$

where $\left.d=\llbracket \log _{4}(3 / R)+1\right]$. Denote by $B_{i}$ the ball with centre $p$ and radius $\left(4^{i+1}+\right.$ $1) R$. Since the support of the kernel of the operator $S_{i}\left(\mathcal{D}_{1}\right)$ is contained in $\{(x, y)$ : $\left.d(x, y) \leq 4^{i+1} R\right\}$ by the finite propagation speed, the function $S_{i}\left(\mathcal{D}_{1}\right) A$ is supported in $B_{i}$.

Now we check that $\mathcal{L} S_{i}\left(\mathcal{D}_{1}\right) A$ is in $\left(Q_{B_{i}}^{k}\right)^{\perp}$. By Proposition 3.3 it suffices to show that $\mathcal{L}^{-k} \mathcal{L} S_{i}\left(\mathcal{D}_{1}\right) A$ is in $L_{0}^{2}\left(B_{i}\right)$. Now, $\mathcal{L}^{-k} \mathcal{L} S_{i}\left(\mathcal{D}_{1}\right) A=S_{i}\left(\mathcal{D}_{1}\right) \mathcal{L}^{1-k} A$ and 
$\mathcal{L}^{1-k} A$ is a constant multiple of a $H^{1}$-atom with support contained in $B(p, R)$ by Remark 3.5. Thus the support of $S_{i}\left(\mathcal{D}_{1}\right) \mathcal{L}^{1-k} A$ is contained in $B_{i}$ and its integral over $M$ vanishes by Proposition 6.6 (ii).

Next, we claim that there exists a constant $C$, independent of $A$, such that for $i$ in $\{0, \ldots, d\}$

$$
\left\|S_{i}\left(\mathcal{D}_{1}\right) A\right\|_{2} \leq C \mu\left(B_{i}\right)^{-1 / 2} 4^{-i}
$$

and

$$
\left\|\mathcal{L} S_{i}\left(\mathcal{D}_{1}\right) A\right\|_{2} \leq C \mu\left(B_{i}\right)^{-1 / 2} 4^{-i} .
$$

Deferring momentarily the proof of the claim, we show that the first inequality in (6.13) follows from it. Indeed, by (6.14) and the triangle inequality,

$$
\|\mathcal{L S} A\|_{X_{\mathrm{at}}^{k}} \leq C \sum_{i=0}^{d}\left\|\mathcal{L} S_{i}\left(\mathcal{D}_{1}\right) A\right\|_{X_{\mathrm{at}}^{k}} .
$$

Now Remark 6.2 and (6.16) imply that

$$
\begin{aligned}
\left\|\mathcal{L} S_{i}\left(\mathcal{D}_{1}\right) A\right\|_{X_{\mathrm{at}}^{k}} & \leq C \mu\left(B_{i}\right)^{1 / 2}\left\|\mathcal{L} S_{i}\left(\mathcal{D}_{1}\right) A\right\|_{2} \\
& \leq C 4^{-i} .
\end{aligned}
$$

Hence

$$
\|\mathcal{L S} A\|_{X_{\mathrm{at}}^{k}} \leq C
$$

as required to prove the first inequality in 6.13).

To conclude the proof of Step II it remains to prove (6.15) and (6.16). The function $S_{0}$ is bounded by Lemma 6.5 hence $S_{0}\left(\mathcal{D}_{1}\right)$ is bounded on $L^{2}(M)$ by the spectral theorem, and

$$
\left\|S_{0}\left(\mathcal{D}_{1}\right)\right\|_{2} \leq\left\|S_{0}\right\|_{\infty}
$$

Since $S_{0}\left(\mathcal{D}_{1}\right) A$ is supported in $B_{0}=B(p, 5 R)$, we have

$$
\left\|S_{0}\left(\mathcal{D}_{1}\right) A\right\|_{2} \leq\left\|S_{0}\left(\mathcal{D}_{1}\right)\right\|_{2}\|A\|_{2} \leq C R^{-n / 2} .
$$

Furthermore, the integral of $S_{0}\left(\mathcal{D}_{1}\right) A$ vanishes by Proposition 6.6 (ii), so that $S_{0}\left(\mathcal{D}_{1}\right) A$ is a constant multiple of an $H^{1}$-atom.

Denote by $k_{S_{i}\left(\mathcal{D}_{1}\right)}$ the integral kernel of the operator $S_{i}\left(\mathcal{D}_{1}\right)$. Observe that

$$
S_{i}\left(\mathcal{D}_{1}\right) A(x)=\int_{B(p, R)} A(y)\left[k_{S_{i}\left(\mathcal{D}_{1}\right)}(x, y)-k_{S_{i}\left(\mathcal{D}_{1}\right)}(x, p)\right] \mathrm{d} \mu(y) .
$$

By Minkowski's integral inequality and the fact that the support of $S_{i}\left(\mathcal{D}_{1}\right) A$ is contained in $B_{i}$, we have that

$$
\begin{aligned}
\left\|S_{i}\left(\mathcal{D}_{1}\right) A\right\|_{2} & =\left\|S_{i}\left(\mathcal{D}_{1}\right) A\right\|_{L^{2}\left(B_{i}\right)} \\
& \leq \int_{B(p, R)}|A(y)| I_{i}(y) \mathrm{d} \mu(y),
\end{aligned}
$$

where

$$
I_{i}(y)=\left\|k_{S_{i}\left(\mathcal{D}_{1}\right)}(\cdot, y)-k_{S_{i}\left(\mathcal{D}_{1}\right)}(\cdot, p)\right\|_{L^{2}\left(B_{i}\right)} \quad \forall y \in B(p, R) .
$$

To estimate $I_{i}(y)$, we observe that

$$
I_{i}(y) \leq d(y, p) \sup _{z \in M}\left\|\mathrm{~d}_{2} k_{S_{i}\left(\mathcal{D}_{1}\right)}(\cdot, z)\right\|_{L^{2}\left(B_{i}\right)},
$$


and, by (6.9) and 6.12 ,

$$
\mathrm{d}_{2} k_{S_{i}\left(\mathcal{D}_{1}\right)}(\cdot, z)=\frac{1}{2 \pi} \int_{-\infty}^{\infty} \phi^{\rho_{i}}(t) P_{N}(\mathcal{O})(\omega \widehat{r})(t) \mathrm{d}_{2} k_{\mathcal{J}\left(t \mathcal{D}_{1}\right)}(\cdot, z) \mathrm{d} t .
$$

Recall that $\phi^{\rho_{i}}$ is supported in $E_{i}=\left\{t \in \mathbb{R}: 4^{i-1} R \leq|t| \leq 4^{i+1} R\right\}$, that the support of $\omega \widehat{r}$ is contained in $[-1,1]$ and that $d(p, y)<R$. Then, by [MMV1, Prop. 2.2 (iii)] (with $\mathcal{J}$ in place of $F$ ), there exists a constant $C$, independent of $i$ and $R$, such that

$$
\begin{aligned}
I_{i}(y) & \left.\leq C d(y, p) \int_{-\infty}^{\infty} \phi^{\rho_{i}}(t) \mid P_{N} \mathcal{O}\right)(\omega \widehat{r})(t) \mid \sup _{z \in M}\left\|\mathrm{~d}_{2} k_{\mathcal{J}\left(t \mathcal{D}_{1}\right)}(\cdot, z)\right\|_{L^{2}\left(B_{i}\right)} \mathrm{d} t \\
& \leq C\left\|t P_{N}(\mathcal{O})(\omega \widehat{r})\right\|_{\infty} R \int_{E_{i}}|t|^{-n / 2-2} \mathrm{~d} t \\
& \leq C R\left(4^{i} R\right)^{-n / 2-1} .
\end{aligned}
$$

Thus,

$$
\begin{aligned}
\left\|S_{i}\left(\mathcal{D}_{1}\right) A\right\|_{2} & \leq C 4^{-i}\left(4^{i} R\right)^{-n / 2}\|A\|_{1} \\
& \leq C 4^{-i} \mu\left(B_{i}\right)^{-1 / 2} .
\end{aligned}
$$

This concludes the proof of (6.15). Now we prove (6.16). Recall that $\mathcal{L}=\mathcal{D}^{2}+b \mathcal{I}=$ $\mathcal{D}_{1}^{2}+\left(b-\kappa^{2}\right) \mathcal{I}$. Therefore

$$
\left\|\mathcal{L} S_{i}\left(\mathcal{D}_{1}\right) A\right\|_{2} \leq\left\|\mathcal{D}_{1}^{2} S_{i}\left(\mathcal{D}_{1}\right) A\right\|_{2}+\left|b-\kappa^{2}\right|\left\|S_{i}\left(\mathcal{D}_{1}\right) A\right\|_{2} .
$$

We first estimate $\left\|\mathcal{D}_{1}^{2} S_{i}\left(\mathcal{D}_{1}\right) A\right\|_{2}$ when $i$ is in $\{1, \ldots, d\}$. Observe that

$$
\mathcal{D}_{1}^{2} S_{i}\left(\mathcal{D}_{1}\right)=\frac{2^{N-1}}{\sqrt{2 \pi}} \int_{-\infty}^{\infty} \frac{\phi^{\rho_{i}}(t)}{t^{2}} P_{N}(\mathcal{O})(\omega \widehat{r})(t) F\left(t \mathcal{D}_{1}\right) \mathrm{d} t
$$

where $F(\lambda)=\lambda^{2} \mathcal{J}(\lambda)$. Since the function $\lambda \mapsto F(t \lambda)$ is an even entire function of exponential type $|t|$ and the support of $\phi^{\rho_{i}}$ is contained in the set $E_{i}$, the support of $F\left(t \mathcal{D}_{1}\right) A$ is contained in $B_{i}$, by finite propagation speed. Thus

$$
F\left(t \mathcal{D}_{1}\right) A(x)=\int_{B(p, R)} A(y)\left[k_{F\left(t \mathcal{D}_{1}\right)}(x, y)-k_{F\left(t \mathcal{D}_{1}\right)}(x, p)\right] \mathrm{d} \mu(y),
$$

and, by Minkowski's integral inequality,

$$
\begin{aligned}
\left\|F\left(t \mathcal{D}_{1}\right) A\right\|_{2} & =\left\|F\left(t \mathcal{D}_{1}\right) A\right\|_{L^{2}\left(B_{i}\right)} \\
& \leq \int_{B(p, R)}|A(y)| I_{i}(y) \mathrm{d} \mu(y) \\
& \leq\|A\|_{1} \sup _{y \in B(p, R)} I_{i}(y),
\end{aligned}
$$

where

$$
I_{i}(y)=\left\|k_{F\left(t \mathcal{D}_{1}\right)}(\cdot, y)-k_{F\left(t \mathcal{D}_{1}\right)}(\cdot, p)\right\|_{L^{2}\left(B_{i}\right)} \quad \forall y \in B(p, R) .
$$

Observe that

$$
I_{i}(y) \leq d(y, p) \sup _{z \in M}\left\|\mathrm{~d}_{2} k_{F\left(t \mathcal{D}_{1}\right)}(\cdot, z)\right\|_{L^{2}\left(B_{i}\right)} .
$$

Since $\sup _{\lambda \in \mathbb{R}^{+}}(1+\lambda)^{N-2}|F(\lambda)|<\infty$ by the asymptotics of Bessel functions of the first kind and $N-2>n / 2+1$ by assumption, we may use [MMV1, Prop. 2.2 (iii)], and conclude that

$$
\sup _{z \in M}\left\|\mathrm{~d}_{2} k_{F\left(t \mathcal{D}_{1}\right)}(\cdot, z)\right\|_{L^{2}\left(B_{i}\right)} \leq C|t|^{-n / 2-1} \quad \forall t \in[-1,1] .
$$


Therefore, since the support of $\phi^{\rho_{i}}$ is contained in the set $E_{i}$,

$$
\begin{aligned}
\left\|\mathcal{D}_{1}^{2} S_{i}\left(\mathcal{D}_{1}\right) A\right\|_{2} & \leq C R \int_{-\infty}^{\infty} \frac{\phi^{\rho_{i}}(t)}{t^{2}}\left|P_{N}(\mathcal{O})(\omega \widehat{r})(t)\right| \sup _{z \in M}\left\|\mathrm{~d}_{2} k_{F\left(t \mathcal{D}_{1}\right)}(\cdot, z)\right\|_{L^{2}\left(B_{i}\right)} \mathrm{d} t \\
& \leq C\left\|t^{-1} P_{N}(\mathcal{O})(\omega \widehat{r})\right\|_{\infty} \frac{R}{\left(4^{i} R\right)^{n / 2+3}} \int_{-\infty}^{\infty} \phi^{\rho_{i}}(t)|t| \mathrm{d} t \\
& \leq C 4^{-i} \mu\left(B_{i}\right)^{-1 / 2} \quad \forall i \in\{1, \ldots, d\} .
\end{aligned}
$$

Now, the inequality (6.16) for $i \in\{1, \ldots, d\}$ follows directly from this, (6.17) and (6.15).

Next we consider $\mathcal{L} S_{0}\left(\mathcal{D}_{1}\right) A$. Observe that $\mathcal{L} S_{0}\left(\mathcal{D}_{1}\right) A$ is supported in $B(p, 5 R)$, and that

$$
\begin{aligned}
\left\|\mathcal{L} S_{0}\left(\mathcal{D}_{1}\right) A\right\|_{2} & \leq\left\|\mathcal{L} S_{0}\left(\mathcal{D}_{1}\right)\right\|_{2}\|A\|_{2} \\
& \leq \mu(B(p, R))^{-1 / 2}\left\|\mathcal{L} S_{0}\left(\mathcal{D}_{1}\right)\right\|_{2} \\
& \leq C \mu(B(p, 5 R))^{-1 / 2}\left\|\mathcal{L} S_{0}\left(\mathcal{D}_{1}\right)\right\|_{2}
\end{aligned}
$$

To prove that $\mathcal{L} S_{0}\left(\mathcal{D}_{1}\right)$ is bounded on $L^{2}(M)$, with norm independent of $R$ in $(0,1]$ observe that, by the spectral theorem and Lemma 6.5

$$
\begin{aligned}
\left\|\mathcal{L} S_{0}\left(\mathcal{D}_{1}\right)\right\|_{2} & \leq \sup _{\lambda \geq 0}\left(\lambda^{2}+b\right)\left|S_{0}(\lambda)\right| \\
& \leq C
\end{aligned}
$$

where $C$ is independent of $R$. This concludes the proof of (6.16), and of Step II.

Step III: proof of the second inequality in (6.13). For each $j$ in $\{1,2,3, \ldots\}$, define $\omega_{j}$ by the formula

$$
\omega_{j}(t)=\omega(t-j)+\omega(t+j) \quad \forall t \in \mathbb{R} .
$$

Observe that $\sum_{j=1}^{\infty} \omega_{j}=1-\omega$ and that the support of $\omega_{j}$ is contained in the set of all $t$ in $\mathbb{R}$ such that $j-3 / 4 \leq|t| \leq j+3 / 4$.

In the rest of this proof, we write $\Omega_{j, N}$ instead of $P_{N}(\mathcal{O})\left(\omega_{j} \widehat{r}\right)$. Observe that the support of $\Omega_{j, N}$ is contained in $\{t \in \mathbb{R}: j-3 / 4 \leq|t| \leq j+3 / 4\}$. Moreover, since $\widehat{r}(t)=c^{-1} \mathrm{e}^{-c|t|}$ and $c>2 \beta$ there exist constants $C, \varepsilon>0$ such that

$$
\left\|\Omega_{j, N}\right\|_{\infty} \leq C \mathrm{e}^{-2 \beta j} \quad \forall j \in\{1,2, \ldots\} .
$$

Define the function $T_{j}: \mathbb{R} \rightarrow \mathbb{C}$ by

$$
T_{j}(\lambda)=\int_{-\infty}^{\infty} \Omega_{j, N}(t) \mathcal{J}(t \lambda) \mathrm{d} t \quad \forall \lambda \in \mathbb{R} .
$$

We may use the observation that $(m-\widehat{\omega} * m)^{\widehat{ }}=\sum_{j=1}^{\infty} \omega_{j} \widehat{m}$ and formula (6.4), and write

$$
\begin{aligned}
(m-\widehat{\omega} * m)(\lambda) & =\frac{1}{2 \pi} \int_{-\infty}^{\infty}(1-\omega(t)) \widehat{r}(t) \cos (t \lambda) \mathrm{d} t \\
& =\sum_{j=1}^{\infty} T_{j}(\lambda) .
\end{aligned}
$$

Then, by the spectral theorem,

$$
\mathcal{T} A=\sum_{j=1}^{\infty} T_{j}\left(\mathcal{D}_{1}\right) A .
$$


Now we estimate $\left\|T_{j}\left(\mathcal{D}_{1}\right) A\right\|_{2}$. By the asymptotics of $J_{N-1 / 2}$ [ , formula (5.11.6), p. 122]

$$
\sup _{s>0}\left|(1+s)^{N} \mathcal{J}(s)\right|<\infty .
$$

By Remark2.4 we may apply [MMV1, Proposition 2.2 (i)], since $N-1 / 2>(n+1) / 2$, and conclude that

$$
\begin{aligned}
\left\|\mathcal{J}\left(t \mathcal{D}_{1}\right) A\right\|_{2} & \leq\|A\|_{1}\left\|\mathcal{J}\left(t \mathcal{D}_{1}\right)\right\|_{1 ; 2} \\
& \leq \sup _{y \in M}\left\|k_{\mathcal{J}\left(t \mathcal{D}_{1}\right)}(\cdot, y)\right\|_{2} \\
& \leq C|t|^{-n / 2}(1+|t|)^{n / 2-\delta} \quad \forall t \in \mathbb{R} \backslash\{0\} .
\end{aligned}
$$

for some $\delta>0$. The function $\mathcal{J}\left(t \mathcal{D}_{1}\right) A$ is supported in $B(p, t+R)$ by Remark 6.7 and has integral 0 by Proposition 6.6 (i). Moreover

$$
\begin{aligned}
\left\|T_{j}\left(\mathcal{D}_{1}\right) A\right\|_{2} & \leq C \int_{-\infty}^{\infty}\left|\Omega_{j, N}(t)\right|\left\|\mathcal{J}\left(t \mathcal{D}_{1}\right) A\right\|_{2} \mathrm{~d} t \\
& \leq C \int_{j-3 / 4}^{j+3 / 4}\left|\Omega_{j, N}(t)\right||t|^{-n / 2}(1+|t|)^{n / 2-\delta} \mathrm{d} t \\
& \leq C \mathrm{e}^{-2 \beta j} \quad \forall j \in\{1,2, \ldots\} .
\end{aligned}
$$

By (2.1) there exist $\varepsilon>0$ such that $\mathrm{e}^{-2 \beta j} \leq C \mu(B(p, j+1))^{-1 / 2} \mathrm{e}^{-\varepsilon j}$. Hence

$$
\left\|T_{j}\left(\mathcal{D}_{1}\right) A\right\|_{2} \leq C \mu(B(p, j+1))^{-1 / 2} \mathrm{e}^{-\varepsilon j} \quad \forall j \in\{1,2, \ldots\} .
$$

Observe that, at least formally,

$$
\mathcal{L} \mathcal{T} A=\sum_{j=1}^{\infty} \mathcal{L} T_{j}\left(\mathcal{D}_{1}\right) A
$$

To prove that the series converges in $X_{\text {at }}^{k}(M)$ we estimate $\left\|\mathcal{L} T_{j}\left(\mathcal{D}_{1}\right) A\right\|_{2}$. Note that

$$
\left\|\mathcal{L} T_{j}\left(\mathcal{D}_{1}\right) A\right\|_{2} \leq\left\|\mathcal{D}_{1}^{2} T_{j}\left(\mathcal{D}_{1}\right) A\right\|_{2}+\left|b-\kappa^{2}\right|\left\|T_{j}\left(\mathcal{D}_{1}\right) A\right\|_{2} .
$$

We have already estimated $\left\|T_{j}\left(\mathcal{D}_{1}\right) A\right\|_{2}$ in (6.21) , so we concentrate on $\left\|\mathcal{D}_{1}^{2} T_{j}\left(\mathcal{D}_{1}\right) A\right\|_{2}$. By (6.20) and the spectral theorem

$$
\mathcal{D}_{1}^{2} T_{j}\left(\mathcal{D}_{1}\right)=\int_{-\infty}^{\infty} \Omega_{j, N}(t) F\left(t \mathcal{D}_{1}\right) \frac{\mathrm{d} t}{t^{2}},
$$

where $F(\lambda)=\lambda^{2} \mathcal{J}(\lambda)$. By using [6.19), [MMV1, Proposition 2.2 (ii)] and the fact that the support of $\Omega_{j, N}$ is contained in $\{t: j-3 / 4 \leq|t| \leq j+3 / 4\}$, we obtain that there exist constants $C$ and $\varepsilon>0$ such that

$$
\begin{aligned}
\left\|\mathcal{D}_{1}^{2} T_{j}\left(\mathcal{D}_{1}\right) A\right\|_{2} & \leq C \int_{-\infty}^{\infty}\left|\Omega_{j, N}(t)\right|\left\|F\left(t \mathcal{D}_{1}\right) A\right\|_{2} \frac{\mathrm{d} t}{t^{2}} \\
& \leq C\|A\|_{1} \int_{-\infty}^{\infty}\left|\Omega_{j, N}(t)\right|\left\|F\left(t \mathcal{D}_{1}\right)\right\|_{1 ; 2} \frac{\mathrm{d} t}{t^{2}} \\
& \leq C \mathrm{e}^{-2 \beta j} \\
& \leq C \mu(B(p, j+1))^{-1 / 2} \mathrm{e}^{-\varepsilon j} \quad \forall j \in\{1,2, \ldots\} .
\end{aligned}
$$


This estimate, (6.21) and (6.22) then imply that

$$
\left\|\mathcal{L} T_{j}\left(\mathcal{D}_{1}\right) A\right\|_{2} \leq C \mu(B(p, j+1))^{-1 / 2} \mathrm{e}^{-\varepsilon j} .
$$

Now, by (6.20) we may write

$$
\begin{aligned}
\mathcal{L} T_{j}\left(\mathcal{D}_{1}\right) A & =\mathcal{L} \int_{-\infty}^{\infty} \Omega_{j, N}(t) \mathcal{J}\left(t \mathcal{D}_{1}\right) A \mathrm{~d} t \\
& =\mathcal{L}^{k} \int_{-\infty}^{\infty} \Omega_{j, N}(t) \mathcal{J}\left(t \mathcal{D}_{1}\right) \mathcal{L}^{1-k} A \mathrm{~d} t \\
& =\mathcal{L}^{k} a_{j},
\end{aligned}
$$

where $a_{j}=\int_{-\infty}^{\infty} \Omega_{j, N}(t) \mathcal{J}\left(t \mathcal{D}_{1}\right) \mathcal{L}^{1-k} A \mathrm{~d} t$.

The function $a_{j}$ is supported in $B(p, j+1)$, since $\mathcal{L}^{1-k} A$ is in $L_{0}^{2}(B(p, R))$ and the kernel of the operator $\int_{-\infty}^{\infty} \Omega_{j, N}(t) \mathcal{J}\left(t \mathcal{D}_{1}\right) \mathrm{d} t$ is supported in $\{(x, y): d(x, y) \leq j\}$. Moreover, $\int_{M} a_{j} \mathrm{~d} \mu=0$ by Proposition 6.6 (ii), and

$$
\left\|a_{j}\right\|_{2} \leq C\left\|\mathcal{L}^{-k}\right\|_{2} \mu(B(p, j+1))^{-1 / 2} \mathrm{e}^{-\varepsilon j},
$$

by (6.23). Hence $a_{j}$ is a multiple of an $H^{1}$-atom supported in $B(p, j+1)$. Then we may apply Lemma 6.1 to the function $a_{j}$, and conclude that $\mathcal{L} T_{j}\left(\mathcal{D}_{1}\right) A=\mathcal{L}^{k} a_{j}$ is in $X_{\mathrm{at}}^{k}(M)$, and that, by (6.23),

$$
\begin{aligned}
\left\|\mathcal{L} T_{j}\left(\mathcal{D}_{1}\right) A\right\|_{X_{\text {at }}^{k}} & \leq C j\left(\mu(B(p, j+1))^{1 / 2}\left\|\mathcal{L} T_{j}\left(\mathcal{D}_{1}\right) A\right\|_{2}\right. \\
& \leq C j \mathrm{e}^{-\varepsilon j} .
\end{aligned}
$$

By summing over $j$, we see that

$$
\|\mathcal{L} \mathcal{T} A\|_{X_{\mathrm{at}}^{k}} \leq C \sum_{j=1}^{\infty} j \mathrm{e}^{-\varepsilon j},
$$

thereby concluding the proof of Step III and of the lemma.

\section{REFERENCES}

[Au] T. Aubin, Espaces de Sobolev sur les variétés Riemanniennes, Bull. Sci. Math., 100 (1976), 149-173.

[ACDH] P. Auscher, T. Coulhon, X.T. Duong and S. Hoffman, Riesz transforms on manifolds and heat kernel regularity, Ann. Sc. Éc. Norm. Sup. 37 (2004), 911-957.

[AMR] P. Auscher, A. McIntosh and E. Russ, Hardy spaces of differential forms on Riemannian manifolds, J. Geom. Anal. 18 (2008), 192-248.

[Br] R. Brooks, A relation between growth and the spectrum of the Laplacian, Math. Z. 178 (1981), 501-508.

[CMM1] A. Carbonaro, G. Mauceri and S. Meda, $H^{1}, B M O$ and singular integrals for certain metric measure spaces, Ann. Sc. Norm. Super. Pisa Cl. Sci., 8 (2009), no. 3, p. 543-582

[Ch] I. Chavel, Riemannian geometry: a modern introduction, Cambridge University Press, 1993.

[CGT] J. Cheeger, M. Gromov and M. Taylor, Finite propagation speed, kernel estimates for functions of the Laplace operator, and the geometry of complete Riemannian manifolds, J. Diff. Geom. 17 (1982), 15-53.

[CW] R.R. Coifman and G. Weiss, Extensions of Hardy spaces and their use in analysis, Bull. Amer. Math. Soc. 83 (1977), 569-645.

[CD] T. Coulhon and X.T. Duong, Riesz transforms for $1 \leq p \leq 2$, Trans. Amer. Math. Soc. 351 (1999), 1151-1169. 
[CMP] M. Cowling, S. Meda and R. Pasquale, Riesz potentials and amalgams, Ann. Inst. Fourier Grenoble 49 (1999), 1345-1367.

[FeS] C. Fefferman and E.M. Stein, $H^{p}$ spaces of several variables, Acta Math. 179 (1972), 137-193.

[He] E. Hebey, Sobolev Spaces on Riemannian Manifolds, Lecture Notes in Mathematics 1635, Springer Verlag, Berlin, 1996.

[L] N.N. Lebedev, Special functions and their applications, Dover Publications, 1972.

[Lo] N. Lohoué, Comparaison des champs de vecteus et des puissances du laplacien sur une variété riemannienne à courbure non positive J. Funct. Anal. 61 (1985), 164-201.

$[\mathrm{MRu}] \quad$ M. Marias and E. Russ, $H^{1}$ boundedness of Riesz transforms and imaginary powers og the Laplacian on Riemanian manifolds, Ark. Mat. 41 (2003), 115-132.

[MMV1] G. Mauceri, S. Meda and M. Vallarino, Weak type 1 estimates for functions of the Laplace-Beltrami operator on manifolds with bounded geometry, arXiv:0811.0104 [math. FA.], to appear in Math. Res. Lett..

[MMV2] G. Mauceri, S. Meda and M. Vallarino, Hardy type spaces on certain noncompact manifolds and applications, arXiv:0812.4209 1 [math.FA].

[MMV3] G. Mauceri, S. Meda and M. Vallarino, Hardy type spaces on noncompact symmetric spaces, in preparation.

[Gr] A. Gry'goryan, Estimates of heat kernels on Riemannian manifolds, in Spectral Theory and Geometry, ICMS Instructional Conference Edinburgh 1988, eds B. Davies and Y. Safarov, London Mathematical Society Lecture Note Series 273, Cambridge University Press, 1999.

[Ru] E. Russ, $H^{1}-L^{1}$ boundedness of Riesz transforms on Riemannian manifolds and on graphs, Pot. Anal. 14 (2001), 301-330.

[St1] E.M. Stein, Topics in Harmonic Analysis Related to the Littlewood-Paley Theory, Annals of Math. Studies, No. 63, Princeton N. J., 1970.

[St2] E.M. Stein, Harmonic Analysis. Real variable methods, orthogonality and oscillatory integrals, Princeton Math. Series No. 43, Princeton N. J., 1993.

[Str] R. S. Strichartz, Analysis of the Laplacian on a complete Riemannian manifold, J. Funct. Anal. 52 (1983), no. 1, 48-79.

[Ta] M.E. Taylor, $L^{p}$ estimates on functions of the Laplace operator, Duke Math. J. 58 (1989), 773-793.

Giancarlo Mauceri: Dipartimento di Matematica, Universitì di Genova, via DodeCANESo 35, 16146 Genova, Italy - MAUCERI@Dima.Unige.IT

Stefano Meda: Dipartimento di Matematica e Applicazioni, Università di MilanoBicocca, via R. Cozzi 53, I-20125 Milano, Italy - stefano.meda@unimib.it

Maria Vallarino: Dipartimento di Matematica e Applicazioni, Università di MilanoBicocCA, via R. CozZi 53, I-20125 Milano, ItAly - MARia.vallaRino@UNimiB.it 